\title{
Granulomatous Slack Skin Disease
}

National Cancer Institute

\section{Source}

National Cancer Institute. Granulomatous Slack Skin Disease. NCI Thesaurus. Code C35464.

A rare variant of mycosis fungoides, characterized by the development of folds of lax skin in the axillae or groins. It has been reported in association with Hodg kin lymphoma. Morphologically, there is a dense granulomatous dermal atypical lymphocytic infiltrate, often associated with multinucleated giant cells. Destruction of elastic tissue is present. Most cases have an indolent clinical course. -- 2005 